\title{
Protective Effect of 2-Deoxy-D-Glucose on Chemotherapeutic Drugs Induced Damages on Peripheral Blood Lymphocytes Exposed in-Vitro
}

\author{
P. Venkatachalam, V.R. Jayanth, Solomon F. D. Paul and V. Vettriselvi \\ Department of Human Genetics, Sri Ramachandra Medical College \& Research Institute \\ (Deemed University), Porur, Chennai 600 116, Tamil Nadu, India
}

KEYWORDS Bleomycin; Mitomycin-C; chromosomal aberrations; sister chromatid exchanges

\begin{abstract}
The effect of 2-deoxy-D-glucose (2-DG), an antimetabolite of glucose was studied in peripheral blood lymphocytes (PBL) exposed to radiomimetic drug bleomycin and an alkylating agent mitomycin-C. The PBL were exposed to 2-DG (5 mM), 30 minutes pretreatment and with Bleomycin $(10$ to $80 \mu \mathrm{g} / \mathrm{ml})$ and Mitomycin-C (2 to12 $\mu \mathrm{g} / \mathrm{ml}$ ) for three hours. The drug as well as 2-DG was removed by washing the cells with HBSS buffer. Then the cells were cultured for 48 hours to study chromosomal aberrations (CA), Translocations (TL) and 72 hours for micronuclei (MN) and Sister Chromatid Exchanges (SCE). Exposures of PBL to Bleomycin and Mitomycin-C showed, a concentration dependent increase in the aberration frequencies, both in the presence and absence of 2-DG. While, the regression analysis showed, that the presence of 2-DG reduced bleomycin induced TL, CA frequencies and Mitomycin$\mathrm{C}$ induced $\mathrm{CA}$ and $\mathrm{MN}$ frequencies significantly $(\mathrm{P}<0.001)$ when compared to $\mathrm{PBL}$ treated with the drugs alone, Bleomycin induced MN frequencies and Mitomycin-C induced SCE's reduction were not significant. The difference could be attributed to the mechanism of the action of drugs on the cells. Furthermore the alteration in the cell cycle kinetics, suggest that the presence of 2-DG during drug exposure, alter the cellular environment and delay the cell proliferation and provide sufficient time to repair the damages, could resulted in the reduced aberration frequencies.
\end{abstract}

\section{INTRODUCTION}

It is well known that most of the radiation used for therapy not only controls the division of tumor cells but also mutagenic to normal cells during multiple cycles of treatment (Bleehen et al. 1974). Therefore, studies were reported on the radio protective effect of compounds like 2Deoxy-D-gluocose (2-DG), Hoechst (Denison et al. 1992) cysteine (Pattet et al. 1949), caffeine (Franchitto et al. 1998) and calcium channel blockers (Rajeeve and Kale 1995) on normal cells. Of which the protective effect of 2-DG was extensively studied in animal models (Jain et al. 1979) and in the lymphocytes of healthy individuals (Kalia et al. 1988; Prabhu et al. 2004) as well as glioma cells followed by radiation (Dwarakanath et al. 1989). In addition to radiotherapy, chemotherapy alone or in combination with radiation plays an important role to treat malignant diseases. The combined use of chemotherapy and radiotherapy, in patients may cause a degree of injury that would

Corresponding Author: Dr P. Venkatachalam

Department of Human Genetics, Sri Ramachandra Medical College \& Research Institute (Deemed University), Porur, Chennai 600 116, Tamil Nadu, India Fax: 91+044-4767008,E-mail: venkip@yahoo.com be clinically toxic following either therapy alone because, both may cause toxic to the same organ by different mechanisms. Furthermore, the response of cellular systems to therapeutic agents depend on its effect on DNA, types of lesions induced, capacity of the cells to repair those lesions and cell cycle kinetics (Jain et al., 1999). As our previous study had shown a protective effect of 2-DG on radiation induced chromosomal aberrations in the PBL (Prabhu et al. 2004), the present study focus on the effect of 2-DG on radiomimectic drug bleomycin and alkylating agent mitomycin-C induced damages in PBL treated in-vitro. The PBL were exposed to those drugs in the presence of 2-DG at $\mathrm{G}_{0}$ phase of the cells, which is the normal stage of majority of the cells in the body at any given time, which enables to some extent that the overall response of tumors surrounding cell to therapeutic drugs. The $5 \mathrm{mM}$ concentration of 2-DG was selected get an equimolar concentration of glucose in the normal blood samples.

\section{MATERIALS AND METHODS}

In-vitro Treatment of Peripheral Blood lymphocytes to Bleomycin and Mitomycin-C: About $10 \mathrm{ml}$ of peripheral blood was collected in a heparinised sterile container from a healthy 
volunteer. The blood was divided into aliquots, suspended in RPMI-1640 medium treated with bleomycin (10 to $80 \mu \mathrm{g} / \mathrm{ml}$ ) or mitomycin-C (2 to12 $\mu \mathrm{g} / \mathrm{ml})$ in the presence and absence of 2-DG (5 $\mathrm{mM}, 30$-min before treatment) for 3 hours at $37^{\circ} \mathrm{C}$. At the end of 3 hours the drugs and 2-DG were removed from the cells by washing with HBSS (Hanks Balanced Salt Solution) buffer for 3 times.

Culture Set up for Chromosomal Aberration: The blood samples treated as mentioned above were used for the preparation of metaphase chromosome for further analysis as described by (Venkatachalam et al. 1999). Briefly, to $1.0 \mathrm{ml}$ of the blood sample, $10 \mathrm{ml}$ culture medium (RPMI1640) supplemented with $7.5 \% \mathrm{NaHCO}_{3}, 20 \%$ fetal calf serum, $200 \mathrm{mM}$ L-Glutamine, penicillin 100 units $/ \mathrm{ml}$ and streptomycin $100 \mathrm{mg} / \mathrm{ml}$, was added. $200 \mathrm{ml}$ of PHA-P was added to the culture to initiate cell division. At 46 hours, the cells were blocked at metaphase stage by adding colcemid at a final concentration of $0.1 \mathrm{mg} / \mathrm{ml}$. The culture was further incubated until 48 hours. The sample was harvested by given hypotonic treatment (20 minutes with $0.45 \% \mathrm{KCl}$ at $37^{\circ} \mathrm{C}$ ), washed thrice with Carnoy's fixative (methanol and acetic acid 3:1) and cast on clean pre-cooled slides. Multiple slides were casted for each sample and used for chromosomal aberration analysis and fluorescence in-situ hybridisation.

To analyse the chromosomal aberrations the slides were stained with $10 \%$ Giemsa, air-dried and mounted with coverslip using DPX. The slides were observed under microscope to record various types of aberrations like dicentric chromosome, ring chromosome, minutes, gaps, acentric fragments and chromatid gaps were recorded as described (Technical report 1986).

Fluorescence in-situ Hybridisation: The slide with metaphase chromosomes prepared as mentioned above was denatured for 6-8 $\mathrm{min}$ in denaturation solution at $70^{\circ} \mathrm{C}$; dehydrated in $70 \%$, $85 \%$ and $100 \%$ ethanol for 2 min each at room temperature and air dried. The whole chromosome probe (\# 2 labelled with TRITC) was mixed with hybridisation buffer and deionised distilled water, denatured at $70^{\circ} \mathrm{C}$ for $5 \mathrm{~min}$ and applied to the slide, containing denatured chromosomes. The slides were sealed with coverslip using rubber cement and hybridisation was carried out for 24 hours at $37^{\circ} \mathrm{C}$ in moistened hybridisation chamber. After 24 hours hybridisation, the coverslip was removed and the slides are rinsed in fromamide wash solution three times, for $10 \mathrm{~min}$ each, $10 \mathrm{~min}$ in $2 \times \mathrm{SSC}$ and 5 min in $2 \times \mathrm{SSC} / 0.1 \% \mathrm{NP}-40$ at $45^{\circ} \mathrm{C}$ sequentially. After air drying the slides were counterstained with DAPI $(7.5 \mu \mathrm{l} / \mathrm{slide})$ and covered with coverslip. The number of painted chromosomes with and without translocation was recorded for each metaphase. The genomic translocation frequency was estimated from the fraction of genome painted as suggested by (Lucas et al. 1992). In case of chromosome-2, it comprises 8.34 $\%$ of genome (Mendelshen et al. 1973), and hence $16.68 \%$ of total exchanges can be detected.

Micronucleus Assay: The blood samples were cultured in RPMI-1640 medium, supplemented with $20 \%$ fetal calf serum and 0.2 ml PHA-M (Paul et al. 1997). Cytochalasin - B at a final concentration of $3 \mu \mathrm{g} / \mathrm{ml}$ culture was added at 44 hours of culture. The cells are further incubated for 28 hours at $37^{\circ} \mathrm{C}$. The cells were harvested with brief hypotonic treatment and slides were prepared by fixing the cells with Carnoy's fixative. The cell suspensions were dropped on to a clear cooled slide and stained with Giemsa. Cells with two daughter nuclei surrounded by cytoplasm were scored for the presence of MN according to criteria of (Paul et al. 1997).

Cell Cycle Kinetics and Sister Chromatid Exchange Assay (SCE): Chromosome culture was set up, as in the case of chromosomal aberration assay, except for the addition of Brdu $(100 \mu \mathrm{l} / 5 \mathrm{ml})$ and the culture time was 72 hours. After the preparation of slides, metaphase chromosomes were stained with Hoechst (33258) for 10 minutes, washed with Mcllvaine's buffer, mounted temporarily with coverslip and exposed to sunlight for 2 hours. The slides were stained with $2 \%$ Giemsa, mounted with DPX and the stages of cell cycle and number of exchanges in the second cell cycle metaphases alone were recorded as per (Technical Report 1986).

Modulatory Factor: The modulatory effect of 2 DG was calculated as given by (Dwarakanath and Jain 1987).

Dose reduction factor $(\mathrm{r})=$

Drug induced aberration frequency with 2-DG

Drug induced aberration frequency without 2-DG

Statistical Analysis: The regression analysis for the aberration frequency was carried out using the SPSS/PC+Software. 


\section{RESULTS}

Aberration Frequency Induced by Bleomycin: Frequencies of TL, CA, and MN obtained from PBL treated with bleomycin in the presence and absence of 2-DG are given in Figures 1-3, respectively. The base line frequencies of TL in the control $(0.046 \pm 0.007)$ are higher than that obtained for $\mathrm{DC}(0.008 \pm 0.002)$ and $\mathrm{MN}(0.005 \pm 0.002)$. It was also observed that bleomycin induced a concentration dependent increase in the frequencies of all types of aberrations. Addition of 2-DG reduced the bleomycin induced TL frequency by $21-60 \%$. Similarly for DC and MN it varies between 21$32 \%$ and $55-84 \%$. The dose reduction factor (DRF) for TL, DC and MN are given in the Table 1. The regression analysis between the aberration frequencies induced by bleomycin with and without 2-DG showed the regression coefficient of $174.2 \times 10^{-2}$ and $0.326 \times 10^{-2}$ for TL, $0.836 \times 10^{-2}$ and $0.230 \times 10^{-2}$ for CA and $0.292 \times 10^{-2}$ and 0.249 $\mathrm{x} 10^{2}$ for $\mathrm{MN}$ for unit increase in the concentration. While, the obtained results showed a significant reduction for TL and CA frequency $(\mathrm{p}<0.001)$ in PBL treated in the presence of 2-DG, the reduction is not significant for MN.

Aberration Frequencies Induced by Mitomycin-C: Frequencies of MN, CA and SCE obtained from PBL treated with mitomycin-C in the presence and absence of 2-DG are given in Figures 4-6, respectively. Similar to bleomycin, mitomycin- $\mathrm{C}$ also induced a concentration dependent increase in the frequencies of all types

Table 1: Dose reduction factor of 2-DG on aberration frequency on peripheral blood lymphocytes exposed to bleomycin

\begin{tabular}{lccc}
\hline $\begin{array}{l}\text { Concentration } \\
(\mu \mathrm{g} / \mathrm{ml})\end{array}$ & $\begin{array}{c}\text { Trans- } \\
\text { locations }\end{array}$ & $\begin{array}{c}\text { Chromosomal } \\
\text { aberrations }\end{array}$ & Micronuclei \\
\hline 10 & 0.47 & 0.21 & 0.55 \\
20 & 0.39 & 0.24 & 0.84 \\
40 & 0.6 & 0.32 & 0.1 \\
80 & 0.21 & 0.27 & 0.83 \\
\hline
\end{tabular}

Table 2: Dose reduction factor of 2-DG on aberration frequency on peripheral blood lymphocytes exposed to mitomycin-C

\begin{tabular}{lccc}
\hline $\begin{array}{l}\text { Concentration } \\
(\mu \mathrm{g} / \mathrm{ml})\end{array}$ & $\begin{array}{c}\text { Sister } \\
\text { chromatid } \\
\text { exchanges }\end{array}$ & $\begin{array}{c}\text { Chromo- } \\
\text { somal } \\
\text { aberrations }\end{array}$ & Micronuclei \\
\hline 2 & 0.42 & 0.51 & 0.86 \\
4 & 0.52 & 0.59 & 0.67 \\
8 & 0.83 & 0.55 & 0.65 \\
10 & 0.78 & 0.54 & 0.73 \\
\hline
\end{tabular}

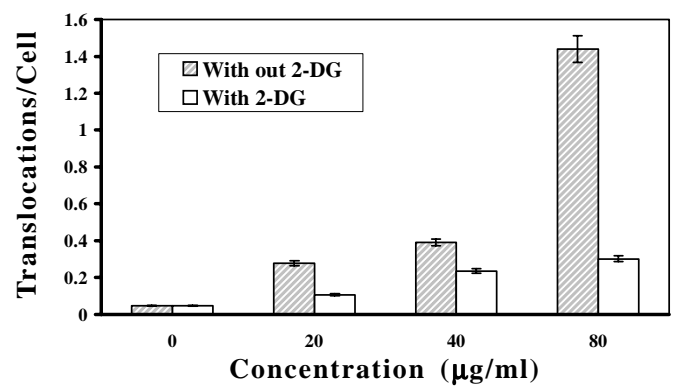

Fig. 1. Frequency of Translocations in peripheral blood lymphocytes exposed to bleomycin

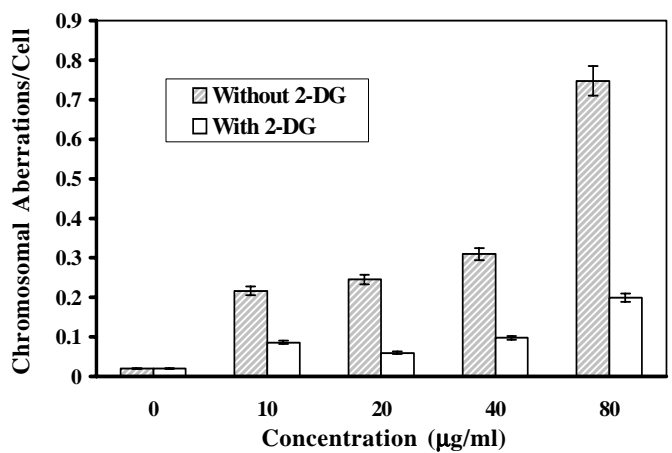

Fig. 2. Frequency of chromosomal aberrations in peripheral blood lymphocytes exposed to bleomycin

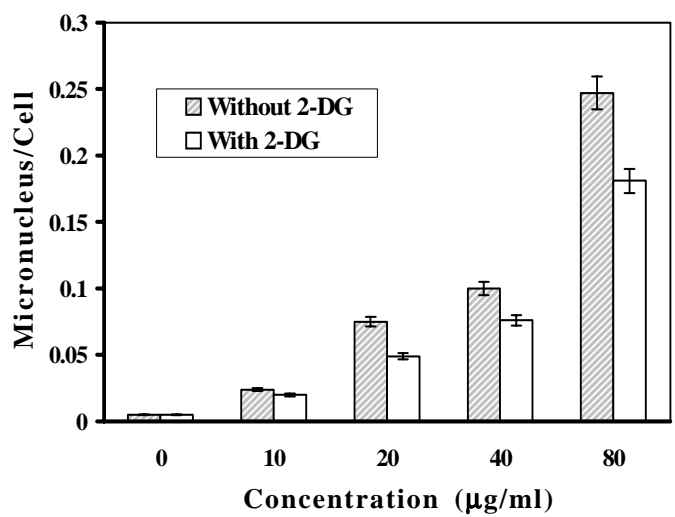

Fig. 3. Frequency of Micronucleus in peripheral blood lymphocytes exposed to bleomycin

of aberrations. Addition of 2-DG reduced the SCE frequency by $42-89 \%$ for various concentrations of mitomycin-C. Similarly for CA and MN it varies between $23-55 \%$ and $65-86 \%$. The dose reduction factor (DRF) for SCE, CA and MN are given in the Table 2 . The regression analysis between the aberration frequencies induced by mitomycin- $\mathrm{C}$ 


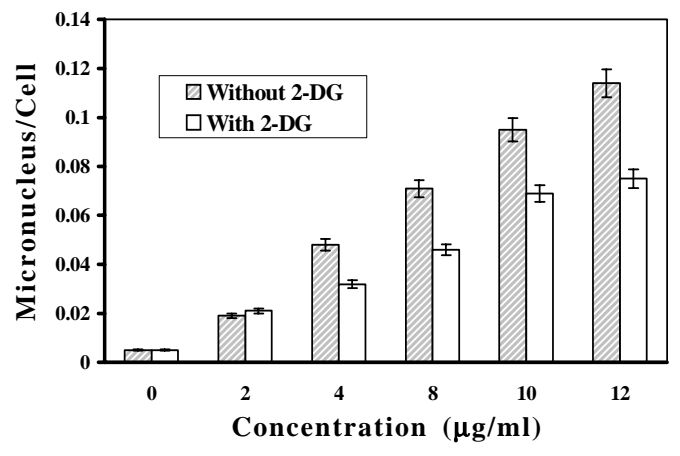

Fig. 4. Frequency of Micronucleus in peripheral blood lymphocytes exposed to mitomycin-C

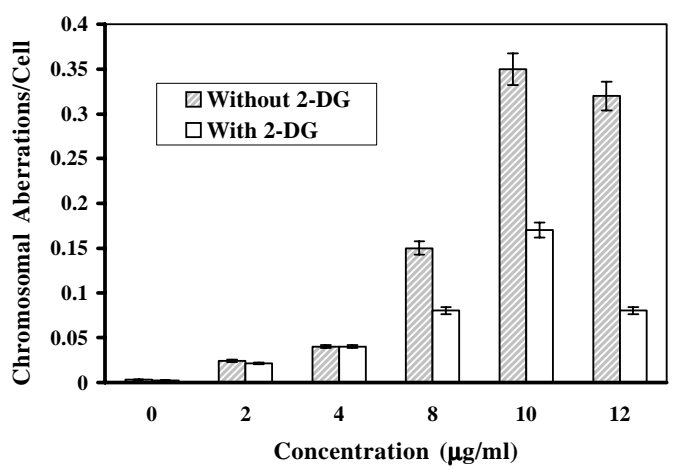

Fig. 5. Frequency of Chromosomal aberrations in peripheral blood lymphocytes exposed to mitomycin-C

with and without 2-DG showed the regression coefficient of $288.2 \times 10^{-2}$ and $272.1 \times 10^{-2}$ for SCE, $2.927 \times 10^{-2}$ and $1.070 \times 10^{-2}$ for CA and $0.889 \times 10^{-2}$



Fig. 6. Frequency of Sister Chromatid Exchanges in peripheral blood lymphocytes exposed to mitomycin-C

and $0.582 \times 10^{-2}$ for $\mathrm{MN}$ for unit increase in the concentration. The results showed that $2-\mathrm{DG}$ reduced the mitomycin- $\mathrm{C}$ induced $\mathrm{CA}$ and $\mathrm{MN}$ frequencies significantly $(\mathrm{p}<0.001)$ and the reduction is not significant for SCE.

Effect of Bleomycin and Mitomycin-C on Cell Cycle Kinetics: Both bleomycin and mitomycin-C reduces proliferation index (PI) and increase the Average Generation Time (AGT). Tables 3 and 4 show the effect of bleomycin and mitomycin-C on cell cycle kinetics. PBL treated with mitomycin and bleomycin, both in the presence and absence of 2-DG showed an increase in AGT and decrease in PI, in a concentration dependent manner. Furthermore, PBL treated with drugs in the presence of 2-DG, the AGT is more when compared with the absence of 2-DG.

Table 3: Cell cycle kinetics in peripheral blood lymphocytes exposed to bleomycin

\begin{tabular}{lcccc}
\hline Concentration & \multicolumn{2}{c}{ Proliferative index $(\%)$} & & \multicolumn{2}{c}{ Average generation time $(\mathrm{h})$} \\
\cline { 2 - 3 }$(\mu \mathrm{g} / \mathrm{ml})$ & With out 2-DG & With 2-DG & & With out 2-DG \\
\hline 0 & 1.15 & 1.06 & 41.7 & 45.28 \\
10 & 1.15 & 1.06 & 41.7 & 45.28 \\
20 & 1.14 & 1.04 & 42.1 & 46.15 \\
40 & 1.12 & 1.0 & 42.9 & 48.00 \\
80 & 1.02 & 1.06 & 47.1 & 45.25 \\
\hline
\end{tabular}

Table 4: Cell cycle kinetics in peripheral blood lymphocytes exposed to mitomycin - C

\begin{tabular}{lccccc}
\hline Concentration & \multicolumn{2}{c}{ Proliferative index $(\%)$} & & \multicolumn{2}{c}{ Average generation time $(h)$} \\
\cline { 2 - 3 }$(\mu \mathrm{g} / \mathrm{ml})$ & With out $2-D G$ & With $2-D G$ & & With out 2-DG & With 2-DG \\
\hline 0.0 & 2.30 & 2.30 & & 31.30 & 31.30 \\
2 & 1.85 & 2.16 & & 38.91 & 33.30 \\
4 & 1.94 & 1.80 & & 37.11 & 41.90 \\
8 & 1.97 & 1.70 & & 36.54 & 52.94 \\
10 & 2.03 & 1.36 & & 35.54 & 45.00 \\
12 & 1.93 & 1.60 & & 37.30 &
\end{tabular}




\section{DISCUSSION}

The damage induced by therapeutic agents as well as mutagens, which leads to the loss of proliferative capacity in mammalian cells, is known to be located in the nucleus. Type of changes in DNA produced during the exposure, depending on the nature of cytotoxic agents. The study on the cytogenetic effects of therapeutic agents is of interest, because, the types of lesions and repair to the DNA are different among individuals and among tissues and response of patients to therapy. As the capacity to repair damages caused by cytotoxic agents is an important characteristic as well cellular system. Repair processes, which act in response to attack by cytotoxic agents profoundly, influence survival, growth, development and transformation of cells. Furthermore all those process are energy dependent events. Our earlier study on the PBL exposed to gamma radiation, showed a reduction in the frequencies of aberrations in the presence of 2-DG (Prabhu et al. 2004). To explore further, the effect of glucose antimetabolite 2-DG on bleomycin and mitomycin-C induced damages on PBL and cell cycle kinetics were studied.

Exposure to both drugs increased chromosomal exchanges, chromatid alterations and MN significantly in all the concentrations studied. Bleomycin induced chromosome as well as chromatid type exchanges, which showed a linear relation to the concentration. Whereas, mitomycin-C induced mainly chromatid type rather than chromosome type exchanges, because of its mechanism of action. Earlier reports showed bleomycin induces predominantly chromosome type aberration in Chinese hamster cells after treated in vitro (Kurten and Obe 1990) and in peripheral blood lymphocytes of treated patients (Schnizel and Schmid 1976). In the present study also, treatment with bleomycin induced predominantly chromosome type aberrations, which was evidenced with $90 \%$ of $\mathrm{DC}$ and rings were associated with fragments as well as translocations. Though the presence of 2-DG, reduces all type of aberrations, only bleomycin induced TL, CA and mitomycin-C induced $\mathrm{CA}$ and $\mathrm{MN}$, showed a significant decrease in all the concentration studied. Similar reduction in the aberration frequencies was observed in normal lymphocytes (Kalia et al. 1988) mice (Jain et al. 1979) and tumor cell lines (Jain et al. 1979; Dwarakanath and Jain 1989) exposed to radiation in the presence of 2-DG. However, the bleomycin induced $\mathrm{MN}$ and mitomycin-C induced SCE frequencies were not reduced significantly of unknown reason. Generally, it is believed that SCE's is a measure of mutagenesis and therefore, 2-DG may not reduce the mutation frequency in normal lymphocytes.

General mechanism proposed for the reduction of damages on normal cells during radiation treatment is (I) Protecting the DNA against the induction of damage, (II) interference with the progression of cells in cell cycle and (III) modifications of repair of damages. It was shown that ATP synthesis and oxidative metabolism were essential for the repair of damages induced by radiation (Dwarakanath and Jain 1989). 2-DG, inhibitor of ATP synthesis through glycolytic pathway in cells, was observed to decrease the manifestation of radiation in yeast and human tumour cells (Jain et al. 1979; 1985). Cellular manifestation of radiation /chemotherapy depends on cellular dynamics like competitive process of DNA repair and fixation of lesions, which might show different energy dependence following treatment. Repair is facilitated under sub-optimal growth conditions and fixation is usually associated with cell proliferation. 2-DG reduces the proliferationlinked fixation of DNA lesions and permits the cells to repair the damages. It was reported that exposure to radiation induce a transient block in cell cycle progression, presumably permit the error free repair of DNA damages before the cell initiate the synthesis or mitosis. Similarly, in the present study also both drugs alter the proliferation kinetics of normal PBL by increasing the AGT. The delay or transient block in cell cycle by the drugs and decreased level of ATP maintain a sub-optimal conditions, favor more error free repair, there by reduction in the damages when cells are exposed to the drug in the presence of 2-DG. Therefore it has been suggested that 2DG in combination with radiotherapy/ chemotherapy could lead an improvement in tumor therapy by inhibiting the repair process and enhance the damage by fixating the lesions.

\section{ACKNOWLEDGEMENTS}

The authors wish to thank the Defense Research Development Organization (DRDO), Government of India and Council of Scientific and Industrial Research (CSIR) for providing the financial assistance to carry our the research work. 


\section{REFERENCES}

Bleehen NM, Gillies E, Twentyman PR 1974. The effect of bleomycin and radiation in combination on mammalian cells in culture. Br J Radiol, 47 :346351 .

Denison L, Haigh A, D'Cunha G, Martin RF 1992. DNA ligants as radioprotectors: Molecular Studies with Hoechest 3342 and Hoechest 33258. Int J Radiat Biol, 61: 69 .

Dwarakanath BS, Jain VK 1989. Energy linked modifications of the radiation response in a human cerebral glioma cell line. Int J Radiation Oncol Biol Phys, 17: 1033-1040.

Dwarakanath BS, Jain VK 1987. Modification of the radiation induced damage by 2 -deoxy-D-glucose in organ cultures of human cerebral gliomas. Int $J$ Radiation Oncol Biol Phys, 13: 741-746.

Franchitto P, Pichierri P, Mosesso F, Palitti F 1998. Cafffeine effect on the mitotic delay induced by G2 treatment with UVC or mitomycin-C. Mutagenesis, 13: 499.

Jain VK, Kalia VK, Gopinath PM, Naqvi S, Kucheria K 1979. Optimization of cancer therapy: Part IIIEffect of combining 2-deoxy-D-glucose treatment with gamma-irradiation on normal mice. Ind J Exp Biol, 20: 1320-1325.

Jain VK, Kalia VK, Sharma R, Maharajan V, Menon M 1985. Effects of 2DG on glycolysis, proliferation kinetics and radiation response of human cancer cells. Int J Radiation Oncol Biol Phys, 11: 943950

Jain VK, Pohlit W, Purohit SC 1975. Influence of energy metabolism on the repair of X-ray damage in living cells IV. Effects of 2DG on the repair phenomena during fractionated irradiation of yeast. Radiat Environ Biophy, 12: 137-142.

Kalia VK, Jain VK, Otto FJ 1988. Optimization of cancer therapy IV: Effects of 2-DG on radiation induced chromosomal damage in PHA stimulated human leucocytes. Ind J Exp Biol, 20: 884-888.
Kurten S, Obe G 1990. Premature chromosome condensation in the bone marrow of Chinese hamster ovary cells after application of bleomycin in vivo. Mutat Res, 228: 157-169.

Lucas JN, Awa AA, Straume T, Poggensee M, Kodama Y, Nakano M, Ohtaki K, Weier HU, Pinkel D, Gray G, Littlefield J 1992. Rapid translocation frequency analysis in human decades after exposure to ionizing radiation. Int $J$ Radiat Biol 62: 53-63.

Mendelshon M, Mayall BH, Bogart E, Moore DH, Benson H, Perry H 1973. DNA content and DNA based centromeric index of the 24 human chromosomes. Science, 179:1126

Pattet HM, Tyree EV, Straube RL, Smith DE 1949. Cystein protection against X-irradiation. Science, 110: 213.

Paul SFD, Venkatachalam P, Jeevanram RK 1997. Analysis of dose-response curve obtained with cytokinesis block micronuclei assay. Nuclear Medicine in Biology 24: 413-416.

Prabhu BK Gowri B Muthuvelu K Venkatachalam P Paul SFD Jayanth VR 2004. Effect of 2DG on the induction of chromosomal aberrations in lymphocytes exposed in-vitro to gamma radiation at a dose rate of $1 \mathrm{~Gy} / \mathrm{minute}$. Int J Hum Genet, 4: 45-49.

Rajeev V, Kale RK 1995. Modulation of radiation induced lipid peroxidation by phospholipase A2 and calmodulin antagonists: Relevance to detoxification. Radiat Phys Chem, 45: 671.

Schnizel W, Schmid W 1976. Lymphocyte chromosome studies in humans exposed to chemical mutagens. The validity of the method in 67 patients under cytostatic therapy. Mutat Res, 40: 139-166.

Technical Report 1986. Biological Dosimetry, Chromosome aberration analysis for dose assessment. In: Technical Report Series No. 260. Vienna: IAEA, pp. 1-69.

Venkatachalam P, Paul SFD, Mohankumar MN, Prabhu BK, Kathiresan A, Jeevanram RK 1999. Higher frequency of dicentrics and micronucleus in peripheral blood lymphocytes of cancer patients. Mutat Res, 425: 1-8. 